\title{
Influence of a channel bend on the purging of saline fluid from a cavity by an overflow of fresh water
}
N. Gillam ${ }^{1}$
S. W. Armfield ${ }^{2}$
M. P. Kirkpatrick ${ }^{3}$

(Received 18 August 2008; revised 6 July 2009)

\begin{abstract}
A numerical and laboratory investigation on the purging of saline fluid from a cavity within a $60^{\circ}$ channel bend is presented to characterise the purging process in open channel bends. The numerical simulation uses a three dimensional, direct numerical simulation, fractional-step solver. The geometry replicates the laboratory experiment to define the mixing processes of a cavity with vertical sidewalls occupying the centre $20^{\circ}$ of the $60^{\circ}$ channel bend. The secondary circulation as the flow moves through the bend increases the vertical momentum exchange within the cavity and hence destabilises the saline interface.
\end{abstract}

\section{Contents}

\section{Introduction}

http://anziamj . austms.org. au/ojs/index.php/ANZIAMJ/article/view/1476 gives this article, (c) Austral. Mathematical Soc. 2009. Published July 14, 2009. ISSN 1446-8735. (Print two pages per sheet of paper.) 
2 Numerical simulation

C992

3 The purging process in a curved channel

C996

4 Conclusion

C1001

References

C1002

\section{Introduction}

Purging of density stabilised pools by an overflow of fresh water in a channel bend is an important fluid dynamics problem crucial to the understanding of environmental flow releases and their associated success in purging saline pools from the bases of rivers within Australia. Saline pools are re-charged primarily through saline groundwater intrusion at the base of the river, and the high salinity and low dissolved oxygen levels render these pools uninhabitable by fish or other aerobic organisms which would otherwise rely on these deeper parts of the river for safe feeding and breeding [1]. During times of no or low flow, the saline groundwater settles within the deepest parts of the river, generally within the scour holes located on river bends. The saline pools generally consist of a very salty bottom layer with a relatively fresh overflowing layer, and with a well defined interface [1]. A sustained flow event is required to purge the density stabilised pool from the base of the river [1], often occurring by way of an environmental release, which is a controlled release of water from managed and collected sources upstream. As a result of the drought, rising consumer demand for the water allocated for such releases means that sound justification of the use of the water for this purpose to be achieved [5].

A numerical and experimental investigation on the purging of a cavity with vertical side walls within the centre of a $60^{\circ}$ open channel bend and occupying the centre $20^{\circ}$ of the bend has been undertaken to identify the large scale 
flow features using an instantaneous start-up, and characterise the purging process in a curved channel. The secondary circulation present, due to the 'centrifugal' acceleration of the flow through the bend, results in a highly 3D flow field within the cavity, and enhances the mixing of saline fluid within the cavity as the fresh overflow is forced into the cavity by the circulation on the outside of the channel bend.

Channel geometry The channel is a narrow open channel with straight inlet and outlet reaches, with a $60^{\circ}$ bend inset, the top view of which is shown in Figure 1. The bend has a centreline radius of curvature of $1 \mathrm{~m}$. The $20^{\circ}$ cavity with vertical side walls is set into the base of the channel in the centre of the bend using false floor inserts, and the height of the cavity, $h_{\text {cav }}$, is $0.13 \mathrm{~m}$.

\section{Numerical simulation}

The numerical simulation solves the 3D non-dimensional Navier-Stokes ( $\mathrm{N}-$ s) Equations (1)-(4) in cylindrical and Cartesian coordinates with the Boussinesq assumption for buoyancy. The Boussinesq assumption has previously successfully been applied to purging in straight channels with strong step transitions in density variation $[3,8]$ where the density difference between the fresh and saline water is small.

The continuity equation is

$$
\frac{\partial u_{r}}{\partial r}+\frac{u_{r}}{r}+\frac{1}{r} \frac{\partial u_{\theta}}{\partial \theta}+\frac{\partial u_{z}}{\partial z}=0 .
$$

The $r, \theta$ and $z$-momentum equations are

$$
\frac{\partial u_{r}}{\partial t}+u_{r} \frac{\partial u_{r}}{\partial r}+\frac{u_{\theta}}{r} \frac{\partial u_{r}}{\partial \theta}+u_{z} \frac{\partial u_{r}}{\partial z}-\frac{u_{\theta}^{2}}{r}
$$




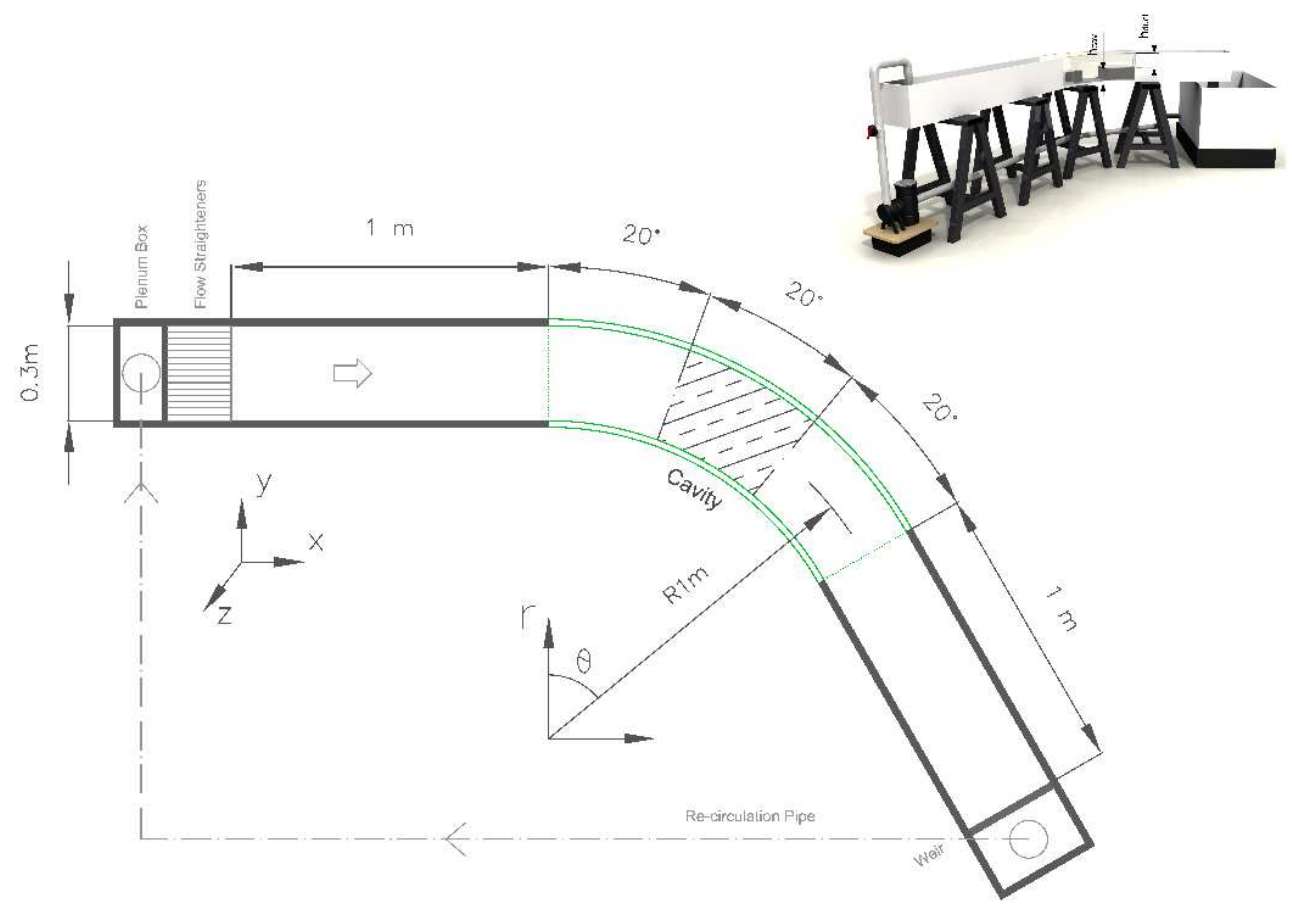

FiguRE 1: Top view of laboratory model replicated by the 3D DNS code and inset 3D CAD representation of the laboratory configuration. The coordinate system is aligned with the centre of curvature of the bend. 


$$
\begin{aligned}
& =-\frac{\partial p}{\partial r}+\frac{1}{\operatorname{Re}}\left[\frac{\partial^{2} u_{r}}{\partial r^{2}}+\frac{1}{r} \frac{\partial u_{r}}{\partial r}-\frac{u_{r}}{r^{2}}+\frac{1}{r^{2}} \frac{\partial^{2} u_{r}}{\partial \theta^{2}}-\frac{2}{r^{2}} \frac{\partial u_{\theta}}{\partial \theta}+\frac{\partial^{2} u_{r}}{\partial z^{2}}\right] \\
& \frac{\partial u_{\theta}}{\partial t}+u_{r} \frac{\partial u_{\theta}}{\partial r}+\frac{u_{\theta}}{r} \frac{\partial u_{\theta}}{\partial \theta}+u_{z} \frac{\partial u_{\theta}}{\partial z}+\frac{u_{\theta} u_{r}}{r} \\
& =-\frac{1}{r} \frac{\partial p}{\partial \theta}+\frac{1}{\operatorname{Re}}\left[\frac{\partial^{2} u_{\theta}}{\partial r^{2}}+\frac{1}{r} \frac{\partial u_{\theta}}{\partial r}-\frac{u_{\theta}}{r^{2}}+\frac{1}{r^{2}} \frac{\partial^{2} u_{\theta}}{\partial \theta^{2}}+\frac{2}{r^{2}} \frac{\partial u_{r}}{\partial \theta}+\frac{\partial^{2} u_{\theta}}{\partial z^{2}}\right] \\
& \frac{\partial u_{z}}{\partial t}+u_{r} \frac{\partial u_{z}}{\partial r}+\frac{u_{\theta}}{r} \frac{\partial u_{z}}{\partial \theta}+u_{z} \frac{\partial u_{z}}{\partial z} \\
& =-\frac{\partial p}{\partial z}+\frac{1}{\operatorname{Re}}\left[\frac{\partial^{2} u_{z}}{\partial r^{2}}+\frac{1}{r} \frac{\partial u_{z}}{\partial r}+\frac{1}{r^{2}} \frac{\partial^{2} u_{z}}{\partial \theta^{2}}+\frac{\partial^{2} u_{z}}{\partial z^{2}}\right]-\frac{\sigma}{F^{2}},
\end{aligned}
$$

respectively. The solute transport equation is

$$
\frac{\partial \sigma}{\partial t}+u_{r} \frac{\partial \sigma}{\partial r}+\frac{u_{\theta}}{r} \frac{\partial \sigma}{\partial \theta}+u_{z} \frac{\partial \sigma}{\partial z}=\frac{1}{\operatorname{ReSc}}\left[\frac{\partial^{2} \sigma}{\partial r^{2}}+\frac{1}{r} \frac{\partial \sigma}{\partial r}+\frac{1}{r^{2}} \frac{\partial^{2} \sigma}{\partial \theta^{2}}+\frac{\partial^{2} \sigma}{\partial z^{2}}\right],
$$

where $u$ is the non-dimensional velocity in the direction specified by the subscript and defined in Figure 1, $t$ is time and $p$ is the pressure. The subscript duct signifies the channel and cav the cavity. The length is nondimensionalised by the height of the channel $h_{\text {duct }}$, the velocity by the overflowing free surface channel velocity $\mathrm{U}_{\infty}$, time by $h_{\text {duct }} / \mathrm{U}_{\infty}$, and the pressure by $\rho U_{\infty}^{2}$. Density is related to non-dimensional solute concentration $\sigma$ by $\rho=\rho_{0}+\sigma \Delta \rho$, where $\rho_{0}=\rho_{\text {duct }}$ and $\Delta \rho=\rho_{\text {cav }}-\rho_{\text {duct }}$. The non-dimensional parameters governing the flow are the Reynolds number, $R e=U_{\infty} h_{\text {duct }} / \nu$, the densimetric Froude number, $\mathrm{Fr}=\mathrm{U}_{\infty} /\left(\Delta \rho \mathrm{gh} \text { cav } / \rho_{0}\right)^{1 / 2}$ and the Schmidt number $\mathrm{Sc}=\boldsymbol{v} / \mathrm{\kappa}$. Here $\mathrm{g}$ is the constant of gravitational acceleration, $\mathrm{\kappa}$ the kinematic diffusivity of salt and $v$ the kinematic viscosity.

The channel has straight inlet and outlet reaches, in which a Cartesian coordinate system is used, and the bend and cavity inset in the base, in which a cylindrical coordinate system is used. Thus the simulation must be capable of resolving the flow field in both coordinate systems. This is accomplished using the following transformations from cylindrical to Cartesian coordinate systems,

$$
\mathrm{r} \Delta \theta=\Delta \mathrm{x}, \quad \Delta \mathrm{r}=\Delta \mathrm{y}, \quad \Delta z=\Delta z
$$


where $r$ is the radial distance from the centre of the bend to the cell centre and $\Delta$ indicates the mesh cell thickness. By setting $r$ to be infinitely large in the straight inlet and outlet reaches, that is $r \rightarrow \infty$, (or in practice $r=$ $10^{100}$ ), the cylindrical $\mathrm{N}-\mathrm{S}$ equations reduce to the Cartesian $\mathrm{N}-\mathrm{S}$ equations, by reducing all curvature source terms to be negligibly small in the Cartesian domain.

The domain, shown in Figure 1, is discretised using a finite volume nonstaggered mesh. The simulation presented uses a mesh of size $350 \times 72 \times 100$ in the $x, y$ and $z$ directions respectively. The mesh is constructed to provide the finest mesh resolution on the duct cavity interface, with $40 \times 72 \times 75$ cells concentrated in the cavity. As the code is in development stages, mesh independence checks have not been completed, and DNS codes cannot accurately capture the thin turbulent small scale flow features apparent during the later stages of purging, as was demonstrated during purging studies in straight channels [8]. Thus, the code is only used at this stage to identify the large scale purging features at low Reynolds numbers during the initial stages of the purging process.

The inlet velocity is specified as a $1 / 7$ th power law profile with a zero salt concentration, while the outlet uses a zero normal gradient outflow boundary condition for velocity and salt concentration. The walls and base of the channel and cavity are no-slip surfaces. The flow in the channel is started impulsively. The solute boundary conditions are zero normal gradients at the surface, base and outlet. The free surface radial elevation is negligible for the Reynolds number simulated, and hence the model is simplified by treating the free surface as a slip boundary condition.

The advective terms in the solute transport Equation (5) are discretised using the SMART scheme [7] and all other derivatives, including the cylindrical source terms, are discretised using second order central schemes. The equations are solved in a segregated manner using a fractional step method. The equations are integrated in time using a Crank-Nicolson time stepping scheme for the diffusive terms and an Adams-Bashforth time stepping scheme 
for the advective terms. A pressure correction equation is solved to provide a pressure update and to enforce the divergence free constraint on the velocity, using a P2 projection approach. This method has been shown to be second order accurate in time [2], and is computationally efficient. The time step is varied dynamically to maintain $0.1<\mathrm{CFL} \leqslant 0.45$. The equations are solved with a Bi-Conjugate Gradient Stabilised solver with a Jacobi preconditioner to increase the rate of convergence [9]. The residuals were $1 \times 10^{-5}$ for mass, momentum and solute concentration, and $1 \times 10^{-7}$ for the pressure correction equation, giving a maximum divergence error of $1 \times 10^{-3}$.

\section{The purging process in a curved channel}

The numerical results are validated by comparison with experimental results. Computational and time limitations mean that at this stage numerical results have only been obtained using $\operatorname{Re}=1,000$ and $\mathrm{Fr}=0.5$ whereas experimental results have been obtained at $\operatorname{Re}=17,300$ and $\mathrm{Fr}=0.5$, all with $\mathrm{Sc}=750$. Therefore it is only appropriate to make a direct comparison for the early stages of the flow, which have been shown to be Re independent [3]. Using an instantaneous start-up, the purging process for this geometry is characterised as follows.

1. Immediately after simulation start-up, the interface tilts with the highest point at the downstream cavity wall, and is lifted high enough to pass out of the cavity in the form of an initial splash, as shown in Figure 2 in the experimental results. This initial splash is attributed to the impulsive start-up of the flow. As seen in straight channels [3, 6, 8], this short lived flow feature removes a significant volume of saline fluid from the cavity, lowering the interface by an order of magnitude more than any other flow feature. The initial splash occurs as a result of potential flow effects, where the streamlines enter the cavity and drive the bulk transport of saline fluid from the cavity, as shown in Figure 3 using the numerical simulation results. The magnitude of the interface 

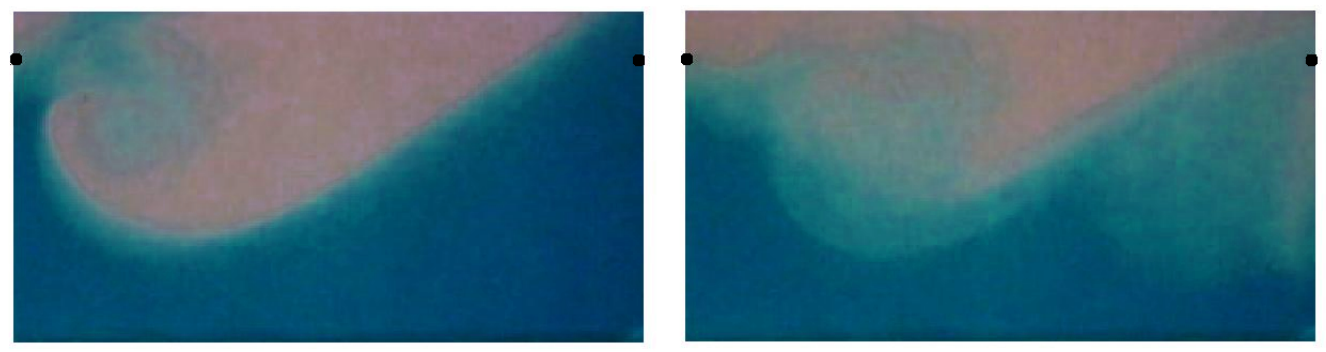

FiguRe 2: Left Image: Stage 1. Initial splash in experimental results at $t=8$. The shed vortex following this initial splash is seen on the upstream (left hand side) edge of the cavity. The cavity is viewed from the centre of curvature of the bend and the saline fluid is dyed blue for visualisation. The black dots indicate the edges of the cavity. Right Image: Stage 2. The shed vortex results in a secondary splash at $t=16$.

tilt is proportional to the Froude number, where the highest Froude number has the largest initial splash [3]. The secondary flow induces a radial variation in the height of the initial splash, lowering the interface further on the outside of the bend than on the inside. The vortex following the initial splash strikes the inside of the bend before the outside and the vortex core which subsequently drops back into the cavity lowers the interface on the outside of the bend further than on the inside.

2. Following the initial splash, a vortex shed from the upstream cavity edge is transported across the cavity, shown in the experimental results in the image on the right in Figure 2 and in the numerical simulation in Figure 4, mixing the saline fluid across the interface and resulting in a secondary splash as this vortex strikes the downstream edge of the cavity. Part of the vortex is not lifted high enough to pass out of the cavity, and this part of the vortex falls back within the cavity and is subsequently transported towards the inside of the bend by the secondary circulation. The vortex drops further into the cavity on the 

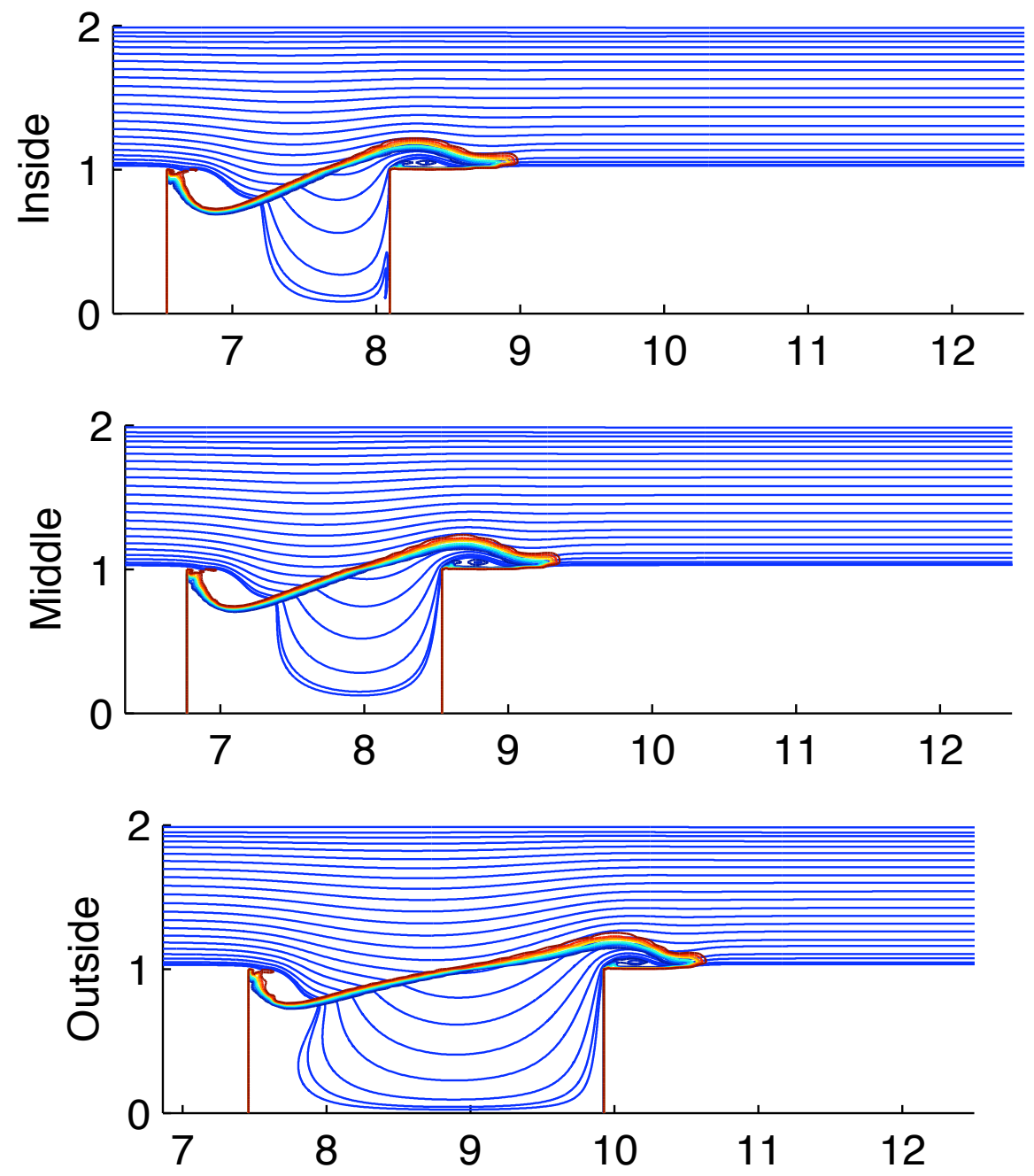

Figure 3: Stage 1. Initial splash using numerical simulation at $t=0.7$. The streamlines enter the cavity and drive the bulk transport of saline fluid from the cavity. Images are plotted along constant radial lines at the inside, or $25 \%$ across the width of the channel from the inner edge, the middle in the centre of the channel, and outside at $75 \%$ across the width of the channel, with streamlines superimposed on solute concentration isolines. There are ten levels of solute concentration levels shown with the lowermost contour representing fully saline fluid. 
outside of the bend than on the inside.

3. Meanwhile, smaller vortices are periodically shed from the upstream cavity edge, mixing the saline fluid across the interface, and are deflected downwards and towards the inside of the bend as they interact with the now fully developed circulatory cell, seen in the experimental results shown in Figure 5. Purging therefore occurs fastest at the downstream cavity wall on the outside of the bend, as both the circulatory cell and the vortices dropping into the cavity erode the interface by pushing it down at this location, baring this cavity bottom region first as shown in Figure 5. The circulatory cell enhances the mixing of saline fluid within the cavity as the fresh overflow is pushed into the cavity on the outside of the bend, replacing the saline fluid. The saline interface on the inside of the bend is consequently lifted above the downstream cavity wall in a corkscrew motion, where it is advected away by the overflow. The majority of saline fluid purged during this stage leaves the cavity from the inside of the channel bend. The volume of saline fluid purged during this stage is therefore influenced by the strength of the secondary circulation. Increasing Re and Fr results in an increased strength of circulation [4], and therefore results in an increased volume of saline fluid purged from the cavity as the circulation de-stabilises the saline interface within the cavity for this geometry. At the low Re and Fr in the numerical simulation, the interface is eroded very slowly by the weak circulation, compared to the experimental flow with high Re and Fr, where purging during this stage occurs very rapidly.

Once the initial and secondary splashes have passed, very little saline fluid is observed to pass out of the cavity on the outside of the bend. The secondary circulation increases the purging rate of saline fluid from the cavity as it permits more saline fluid to be mixed with the fresh overflow, and creates a strongly 3D flow field. During the later stages of purging, the interface is eroded by turbulent eddies across the interface, and the streamers ejected from the waves are extremely thin. These small scale flow features are not 

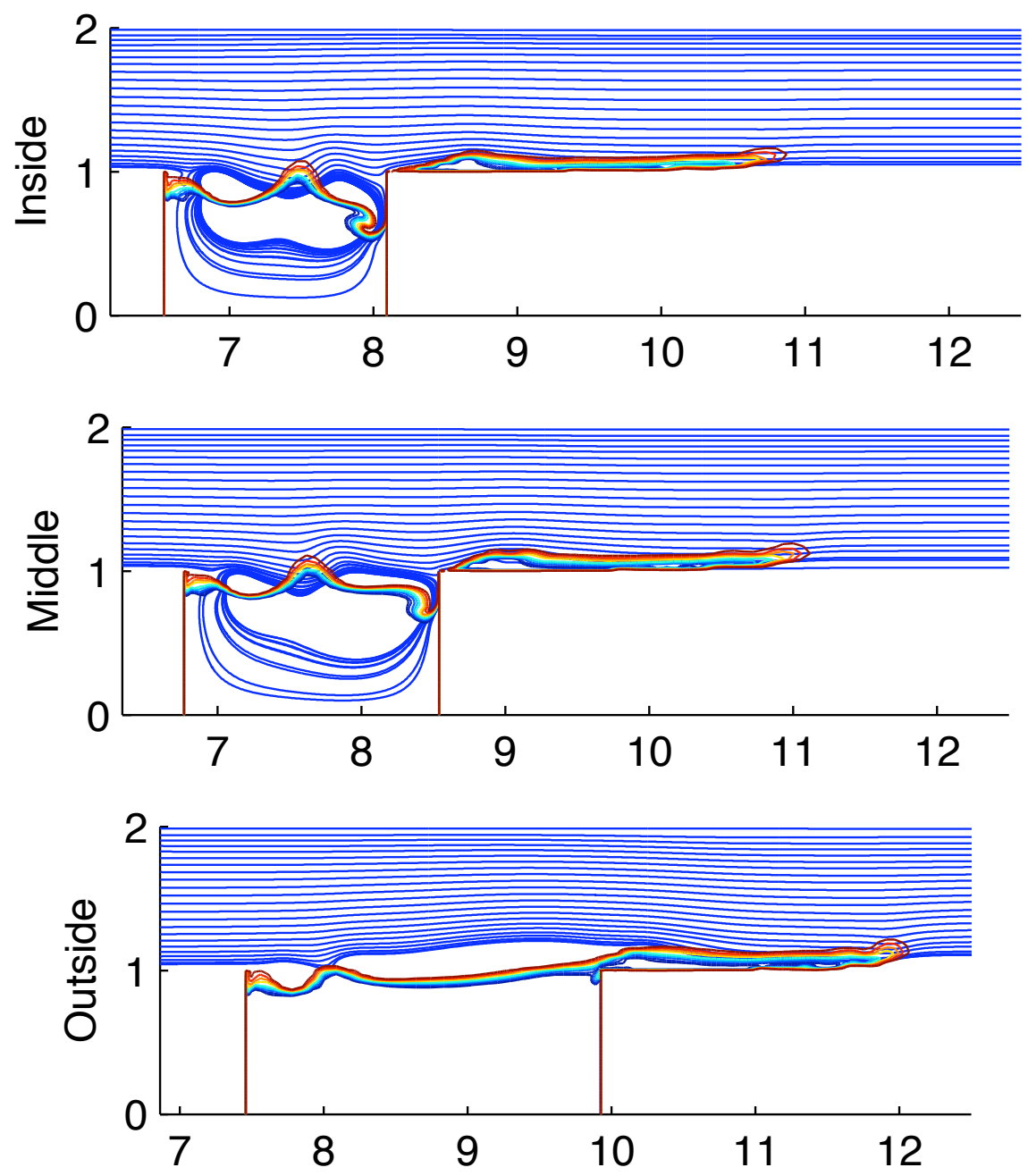

Figure 4: Stage 2. A vortex following the initial splash results in a secondary splash at $\mathrm{t}=2$. 

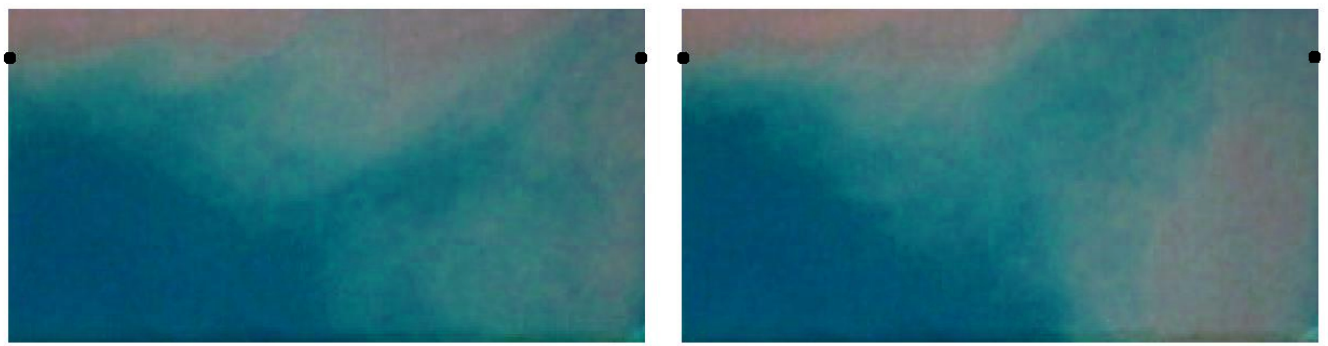

Figure 5: Stage 3. Small vortices are periodically shed from the upstream cavity corner, interacting with the secondary circulation and resulting in a complex 3D flow field. The left image is $t=22$ and the right $t=26$. The secondary circulation bares the downstream cavity corner on the outside of the bend first.

examined in this article. These small scale flow features cannot be captured on a feasible grid scale using DNS simulations, and thus the purging process during this stage will be under predicted by the DNS simulation. As previously seen in straight channels during these later stages [8], DNs is unable to reproduce all of the complex flow interactions and a Large Eddy Simulation turbulence model is required to represent the affect of all of these flow features.

\section{Conclusion}

A numerical code has been developed in order to characterise the purging process in a curved $60^{\circ}$ open channel bend. The centrifugal effects as the overflow moves through the bend drives the transport of saline fluid from the cavity, as this fresh overflow is pushed down into the cavity on the outside of the bend, de-stabilising the dense interface and increasing the mixing of saline fluid within the cavity as the circulatory cell is directed towards the inside of the channel bend. Once the initial splash has passed, saline fluid leaves 
the cavity only on the inside of the bend, as the secondary circulation lifts the saline interface at this location. The later stages of the purging process is driven by a complex interaction of shed vorticity from the upstream cavity corner and the secondary circulation across the width of the channel.

Acknowledgements The first author thanks the Wimmera Catchment Management Authority in Horsham, Victoria, for access to field data. This research was supported by the Australian Research Council in the form of a Joint ARC Linkage project (LP0562390) undertaken with the Victorian Environmental Protection Authority, The University of Sydney and Griffith University.

\section{References}

[1] J. R. Anderson and A. K. Morrison. Environmental consequences of saline groundwater intrusion into the wimmera river, victoria. $B M R$. Journal of Australian Geology and Geophysics, 11:233-235, 1989d. C991

[2] S. Armfield and R. Street. An analysis and comparison of the time accuracy of fractional-step methods for the navier-stokes equations on staggered grids. International Journal for Numerical Methods in Fluids, 38(3):255-282, 2002. C996

[3] S. W. Armfield and W. Debler. Purging of density stabilized basins. International Journal of Heat and Mass Transfer, 36(2):519-530, January 1993. C992, C996, C997

[4] J. C. Bathurst, C. R. Thorne, and R. D. Hey. Secondary flow and shear stress at river bends. Journal of the Hydraulics Division, ASCE, 105 (HY10):1277-1295, 1979. C999

[5] H. Christie. Real-life management of an environmental water reserve, a wimmera perspective. In A.L. Wilson, R.L. Dehaan, R.J. Watts, K.J. 
Page, K.H. Bowmer, and A. Curtis, editors, Proceedings of the 5th Australian Stream Management Conference. Australian rivers: making a difference. Charles Sturt University, Thurgoona, New South Wales., 2007. C991

[6] W. Debler and S. W. Armfield. The purging of saline water from rectangular and trapezoidal cavities by an overflow of turbulent sweet water. Journal of Hydraulic Research, 35:43-62, 1997. C996

[7] P. H. Gaskell and A. K. C. Lau. Curvature-compensated convective transport: Smart, a new boundedness- preserving transport algorithm. International Journal for Numerical Methods in Fluids, 8(6):617-641, 1988. C995

[8] M. P. Kirkpatrick and S. W. Armfield. Experimental and large eddy simulation results for the purging of salt water from a cavity by an overflow of fresh water. International Journal of Heat and Mass Transfer, 48(2):341-359, January 2005. C992, C995, C996, C1001

[9] Henk A. van der Vorst. Iterative solution methods for certain sparse linear systems with a non-symmetric matrix arising from pde-problems. Journal of Computational Physics, 44(1):1-19, November 1981. C996

\section{Author addresses}

1. N. Gillam, School of AMME, University of Sydney, NSW, Australia.

mailto:n.gillam@usyd.edu.au

2. S. W. Armfield, School of AMME, University of Sydney, NSW, Australia.

3. M. P. Kirkpatrick, School of AMME, University of Sydney, NSW, Australia. 